\title{
Improvised desirability function for dual response surface
}

\begin{abstract}
Quality engineering practitioners have great interest for using response surface method in a real situation. Recently, dual response surface has been widely used extensively and is known as one powerful tool for robust design. However, existing methods do not consider the information provided by customers and design engineers. One of the methods that can be used to simultaneously optimize multiple responses is by using the desirability function technique. In this technique, the desirability function approach uses a dimensionality reduction approach that converts multiple predicted responses into a single response problem. The traditional procedures construct the process location and process scale based on sample mean and sample variance respectively. Then, for the regression fitting, the Ordinary Least Square (OLS) method is usually used to acquire the sufficient response functions for the process location and scale based on mean and variance. Nevertheless, these existing procedures are easily influenced by outliers. As an alternative, we propose an improvised desirability function for dual response (IDFDR) to rectify this problem. A numerical example is presented to assess the performance of the IDFDR method. The numerical results signify that the IDFDR method is more efficient than the existing methods.
\end{abstract}

Keyword: Dual response; IDFDR; Outlier; Robust design 